\title{
EEG/QEEG technology identifies neurobiomarkers critical to medication selection and treatment in refractory cases
}

\begin{abstract}
In psychiatry, the reliability of diagnosis (a symptom-based approach) is instrumental in the management of medications and treatments. The National Institute of Mental Health (NIMH) is now searching for neurobiological measures that account for observed and reported symptoms. The results of our study of 386 refractory clinical cases suggest there are four neurobiological measures that account for medication failure: encephalopathy, focal slowing, beta spindles and transient discharges. One or more of these neurobiological measures (neurobiomarkers) explained why medication failed in each of these refractory cases. Our investigation found positive correlations between numbers of medications being prescribed and numbers of neurobiomarkers being identified in children and adolescents. In adults we also we found a positive correlation between numbers of medications being prescribed and number of diagnoses. This study represents a model that could improve the efficacy of the psychotropic intervention and treatment selection in refractory psychiatric cases.
\end{abstract}

Keywords: EEG, QEEG, biomarker, neurobiomarkers, psychotropic drugs, pharmacoEEG
Volume I Issue 7 - 2014

\author{
Ronald J Swatzyna,' Jay D Tarnow, ${ }^{2}$ Jonika D \\ Tannous, ${ }^{3}$ Vijayan Pillai, ${ }^{4}$ Christine Schieszler, ${ }^{5}$ \\ Gerald P Kozlowski ${ }^{6}$ \\ 'Director of Electrophysiological Research, Tarnow Center for \\ Self-Management, Texas \\ ${ }^{2}$ Director for Tarnow Center for Self-Management, Texas \\ ${ }^{3}$ Rice University, Tarnow Center for Self-Management, Texas \\ 4The University of Texas at Arlington Graduate School of Social \\ Work, Texas \\ ${ }^{5}$ The University of Houston Graduate College of Social Work, \\ Tarnow Center for Self-Management, Texas \\ 6Department of Clinical Psychology, Saybrook University, \\ Canada
}

Correspondence: Ronald J Swatzyna, Director of Electrophysiological Research, Tarnow Center for SelfManagement, 1001 West Loop South, Suite 215, Houston, 77027, Texas, USA, Tel 7|3-62|-95I5, Fax 7|3-62|-70|5,

Email drron@tarnowcenter.com

Received: August II, 20I4 | Published: December 06, 2014

\section{Introduction}

Galderisi and Sannita $^{1}$ noted in their prescient review that Pharmaco-EEG was a promising area which could improve psychotropic intervention using neurological data. As they pointed out this intervention has failed to make its way into mainstream psychiatry. Yet, the field of psychiatry still desires evidence-based guidance for selecting and titrating medications more now then ever. Currently psychiatry uses a trial and error process called: "Single Clinical Trials." Physicians with many years of experience have their extensive backgrounds to guide them. Young psychiatrists with only five to fifteen years of experience often lack the expertise. To tackle this growing demand, we present a neurobiomarker model for use in the clinical setting based on the science of Pharmaco-EEG and our study of 386 clinical cases.

Psychiatry does well in many instances: however there are some perplexing refractory cases that do not respond well to traditional psychotropic intervention. Typical psychiatric practice involves a thorough evaluation. Using the Diagnostic and Statistical Manual-5 (DSM-5), a phenomenological based system, psychiatrists make diagnoses. Based on the diagnoses and his/her clinical judgment, they prescribe the appropriate medication. Occasionally patients return complaining of unimproved symptoms and/or intolerable side effects. The psychiatrist may than refer these patients for psychological testing to further define his/her diagnosis. Despite the reliability of psychological testing to more clearly define a diagnosis, it does not explain the reasons behind the medication failure. It is important to note that, in such refractory cases, there are likely confounding neurobiological causes that account for repeated medication failure and/or iatrogenic side effects.
In early 2013, the National Institute of Mental Health (NIMH) launched the Research Domain Criteria (RDoC), which is an effort to evolve the diagnostic process by incorporating a multidisciplinary approach that relies not only on symptoms, but also on genetics, neuroimaging, and cognitive science. This movement away from the use of DSM categorization towards a biological based classification highlights the importance of psychiatry exploring the potential of available electrophysiological testing. Clinical electroencephalograms (EEG) and quantitative EEG (qEEG) technologies have existed for over four decades, yet their application has been very limited in psychiatry. Some studies suggest effective application of these in the diagnosis, medication response, and treatment selection. ${ }^{2}$

An EEG provides important clinical-electrical information by identifying the presence and morphology of critical neurobiomarkers in the raw data. Neurobiomarkers will be used here to indicate abnormal fluctuations in an EEG and the output of the qEEG. Many abnormalities seen in the EEG are considered normal variants in the general population; however, minor abnormalities are important when clinical correlation exists. ${ }^{3,4}$ Clinical correlation is characterized by impaired functioning that corresponds with neurological abnormalities specific to the region of the brain responsible for those processes. Equally as important as the EEG is the qEEG, which compares the EEG data to a normative database and quantifies the significance of the anomalies identified in the EEG. Studies confirmed the usefulness of the qEEG in the diagnostic process when coupled with behavioral testing and clinical evaluation. Preliminary data evidence suggests qEEG accuracy provides sufficient evidence for prescribing appropriate medication during initial treatment. ${ }^{2}$ In 2013, research acknowledged the usefulness of non-invasive techniques that allow highly precise measurement of brain functioning, particularly the 
qEEG. This technology is underutilized in general neurology practice and has promising potential for clinical application. ${ }^{5,6}$

It is commonly accepted that there is a direct and reciprocal relationship between brain function and behavior. Parallel to this, the capability to detect neuronal irregularities may provide insight for the appropriate diagnosis of psychiatric disorders. ${ }^{7}$ The process of neurobiomarker profiling utilizes EEG, qEEG, and clinical presentation to identify neurobiomarkers that may account for medication failure and subsequent treatment difficulties and successes. Beginning in 2009, investigators started using the neurobiomarker profiling model to assist physicians with cases that did not respond as expected to prescribed medications. As the profiling continued, it was noted that there were four emerging neurobiomarkers in refractory cases: encephalopathy, focal slowing, beta spindles, and transient discharges.

Encephalopathy (EN) can be defined as a diffuse disturbance in brain function producing neurological and psychological manifestations. According to Yamanda and $\mathrm{Meng}^{3}$ there are at least 81 different causes of encephalopathy including metabolic disorders, vitamin deficiencies, endocrine diseases, degenerative disorders, inflammatory/infectious diseases, dementia and others. When diffuse encephalopathy is identified, the medical problem should first be addressed before attempting neurologic/psychological treatment. ${ }^{8}$ Medications may have limited the success if the medical problem is not resolved first.

Focal Slowing (FS) is characterized by a predominance of slower activity in a particular area of the brain. Common causes are underlying cortical lesions or tumors.,9 Medications are excellent at adjusting diffuse tuning in the brain. However, there are no medications approved by the Food and Drug Administration (FDA) that adjust focal deficits in the brain. In attempts to use medication designed to target the frequency of the whole brain for treatment of focal issues, adverse side effects are commonly experienced.

Beta spindles (BS) are identified as synchronous activity in the beta range around a specific frequency and are indicative of hyper-arousal. Frederick and Gibbs ${ }^{10}$ identified beta spindling as a component of epilepsy in the 1930's. Beta spindles have also been recognized in Bipolar Disorder, Autism, Obsessive-Compulsive Disorder, some forms of anxiety and Attention Deficit Hyperactive Disorder. ${ }^{11,12}$ Therefore, medications that increase beta where endogenous beta spindles exist are contraindicated and would result in greater risk for cortical irritability.

Transient discharges (TD) are defined as "EEG cerebral dysrhythmias identified by isolated episodic paroxysmal bursts of slow activity, controversial/anomalous spiky waveforms and/or true non-controversial epileptiform discharges". ${ }^{13}$ Transient discharges have been identified in persons with schizophrenia, criminal behavior, violence and disorders of mood, anxiety, panic, obsessive-compulsive, eating and personality; and psychogenic non-epileptic seizures. ${ }^{13}$ Detecting transient discharges can also aid in the identification of cerebrovascular disorders. ${ }^{4}$ If there is clinical correlation at the location of the TD, the EEG would prove to be an effective technique for detection of a psychiatric problem. Medications that lower seizure threshold would likely contribute to the abnormal activity. There should be a significant association between psychiatric diagnosis and neurobiomarkers. Also, as the number of diagnoses and medications increase, a higher number of neurobiomarkers should be identified.

\section{Method}

\section{Dataset}

The data was obtained from the Tarnow Center for SelfManagement (Houston, TX) EEG/qEEG database. The database contained demographic information, diagnosis, neurobiomarkers and the number of medications prescribed for 386 clinical cases. The individuals were divided into three age groups: $5-11,12-17$ and 18 - 69. The IRB of The University of Texas at Arlington deemed that this study is exempt from IRB approval since no identifiable patient information was required for data analysis by the investigators thereby protecting the confidential rights of all patients. The Tarnow Center practices all HIPAA regulations.

\section{EEG Data Collection Equipment}

A Deymed TruScan 32 was used to record the EEG's of the participants. Linked ears and averaging montages were referenced according to the International 10/20 System.

\section{Design}

There were four presenting diagnoses in this sample of sufficient numbers for statistical analysis: Attention Deficit Disorder (ADD), Autism Spectrum Disorders (ASD), Major Depressive Disorder (MDD) and Anxiety Disorders (ANX). Table 1 identifies the numbers of children, adolescents, and adults with each diagnosis. Many of the subjects exhibited comorbidity. As mentioned preciously the neurobiomarkers identified in this study were focal slowing (FS), beta spindles (BS), encephalopathy (EN) and transient discharges (TD). Table 2 identifies the numbers of children, adolescents, and adults with each neurobiomarker. Some subjects exhibited more than one neurobiomarker. The relationship between the diagnoses and neurobiomarkers was examined separately for each age group. Interrater-reliability was assured by having the same team does all of the artifact and conversion of the data to topographical brain maps. Similarly, the same encephalographer and qEEG diplomat wrote reports on all 386 cases. Lastly, the clinical director onsite excluded all cases where clinical correlation was not found. For example TDs are considered normal variants; however, if the TD in left posterior temporal area correlated with expressive aphasia, then it would be included. ${ }^{4}$

\section{EEG Data Acquisition and Processing}

Researchers obtained written informed consent from the participants. The EEG electro-caps were used to collect the EEG data and Electro gel ${ }^{\mathrm{TM}}$ was applied to the 19 sites (Fp1, Fp2, F7, F3, Fz, F4, F8, T3, C3, Cz, C4, T4, T5, P3, Pz, P4, T6, O1 and O2) until impedances were at $<5 \Omega$. The researchers transferred demographic information, patient diagnoses and medication history to a master Exceltm spreadsheet for the study. The participants were recorded resting for a minimum of 10minutes with their eyes open and 10 minutes with their eyes closed and were given the choice of which condition came first. Participants were also permitted to take mental breaks throughout to prevent restlessness that might obscure the recording. The recordings were paused and then resumed when the participant was ready. Following the session, the EEG went through the process of artifact, and qEEG topographical brain mapping at the Human Brain Institute in Saint Petersburg, Russia. A Board Certified electroencephalographer identified any abnormalities in the raw EEG. A qEEG Diplomat analyzed all the qEEG's. All 386 were individual clinical cases. No repeat EEG's or qEEG's were included. 
Table I Diagnoses and numbers in each population

\begin{tabular}{lllll}
\hline & ADD & ASD & DEP & ANX \\
\hline Children & 71 & 21 & 29 & 41 \\
Adolescents & 59 & 25 & 17 & 30 \\
Adults & 96 & 35 & 51 & 55 \\
\hline
\end{tabular}

Table 2 Neurobiomarker numbers in each population

\begin{tabular}{lllll}
\hline & EN & FS & BS & TD \\
\hline Children & 19 & 19 & 21 & 36 \\
Adolescents & 19 & 19 & 20 & 25 \\
Adults & 24 & 24 & 46 & 93 \\
\hline
\end{tabular}

\section{Clinical Correlation Acquisition Process}

Each patient in the study had failed on at least two medications and was referred by a psychiatrist who provided the diagnosis using the DSM-IV (Diagnostic and Statistical Manual of Mental Disorders, fourth edition) criteria. To provide consistency of clinical presentation, each patient (or parent if the patient was $<14 \mathrm{y} / \mathrm{o}$ ) completed a 300item Likert-type scaled set of questions designed to identify their clinical symptoms. It was important to note, neurobiomarkers became a variable in the patient's dataset only when:

a) Abnormalities (even minor deviations) were identified in the raw EEG.

b) These abnormalities reached a level of significance expected in the qEEG.

c) The patient identified issues in the same area.

\section{Results}

The correlations between the number of neurobiomarkers and the number of medication prescribed were assessed for various age groups. Since this involves a number of age group comparisons, significance testing of the correlations necessitates adjustments to the $\mathrm{P}$ values through the use of corrections such as the Bonferroni. Alternatively, the correlations may be presented along with their confidence intervals estimated through resampling methods. ${ }^{14}$ In this study, we present the bootstrap estimates of the correlations and associated confidence intervals. For children, a positive bootstrap estimate of correlation ( $\mathrm{r}$ $=0.080$ ) was found between total neurobiomarkers and the number of medications prescribed. In adolescents, $r=0.220, p=0.015,95 \%$ CI $0.032,0.424$, there was a positive bootstrap estimate of correlation between total neurobiomarkers and medications prescribed. In adults, the relationship between total diagnoses and number of medications prescribed was significant, $r=0.176, p=0.030,95 \%$ CI $0.176,0.034$.

\section{Discussion}

We hypothesized that patients with multiple diagnoses taking multiple medications would likely have more neurobiomarkers identified in their EEG/qEEG's. In children and adolescents we found a positive correlation between number of neurobiomarkers and the number of medications they were taking. This finding supports the difficulty psychiatry has when multiple neurological abnormalities exists. In adults, we found a positive correlation between the total number of diagnoses and number of medications. So the data suggests that as comorbidity increases, so does the number of medications being prescribed.

\section{Limitations}

In the ideal situation, it would be preferable that all participants be medication free prior to the EEG. However, in many cases it was not advisable to remove medications from refractory patients in an out-patient setting. In this study most of the participants were actively taking medications prior to the EEG, which may alter the results and the outcome of the study. Medication results were taken into account for each participant. For example, we ruled out sedative medication effects in cases where BS was identified. Since this study was a clinical sample, a few things could not be standardized, including: time of day, eyes open or closed first during the qEEG, mental breaks and last time the participant had a meal. However, the researchers determined that if the client chose the time of day for their appointment, which qEEG condition was completed first and if they needed a mental break would result in an EEG with fewer artifacts. We regard 386 patients as a suitable number for the study. However, a larger sample size is always desirable.

\section{Future directions}

Ideally the study would have separated the children and adolescent study from the adult study. We found more utility of neurobiomarker profiling in neuroatypical children. To allow for outcome comparison, patients would be randomly assigned to either a traditional treatment group or the neurobiomarker profiling group. This technology could be easily validated in studies that would assess the impact of medications over time, especially in children and adolescent populations; two populations that are especially vulnerable during neurological development. Therefore, researchers should also examine the long term effects of medications.

A better way to test the predictive power of the neurobiomarker profiling process would be to use a pre-test/post-test design with random selection assigning patients to either a control group using typical psychiatric approaches or an experimental group using neurobiomarker profiling. This design will allow researchers to have a baseline group to compare the success rate of medications based on the original diagnoses. Another future direction would be to apply this technique to individual disorders to study the reliability and validity of neurobiomarker profiling for each disorder. The present DSM system uses a phenomenological system. The neurobiomarker model uses a system based on biology. Neurobiomarkers, genetic and genotypic investigations are the direction in which psychiatry needs to go as noted by Thomas Insel ${ }^{15}$ of the NIMH. Studies are underway by investigators funded by the NIMH with the investment by the government as proposed by President Obama's Executive Order National Research Action Plan. ${ }^{16}$

\section{Conclusion}

Our study was twofold, to identify the neurobiomarkers responsible for medication failure in refractory cases and to develop a model the treatment of these difficult cases. We learned that when used separately, an EEG, a qEEG and clinical presentation failed to provide assistance with refractory cases. However when all three were combined, the shortcomings of each is minimized by the group as a whole. This study used surface EEG and the quantitative analysis of the data integrated with a comprehension of neuro function so the focus was not just on a statistical frequency but the neural networks and the neurotransmitters involved.

It was not just the electrical event being identified; it was the comprehension of the underlying biology that was involved as well. If unidentified, EN, FS, BS, and TD can thwart any true improvement in psychiatric health. When examining refractory cases in which psychiatric medications have failed, we deduced that patients were likely to have one or more of these four neuro-biomarkers. The existence of these neuronal irregularities appeared to reflect 
indisputable evidence of brain dysfunction that affect psychiatric pathologies..$^{13}$

\section{Disclosure of conflicting interest}

The authors declared no potential conflicts of interest with respect to the research, and/or publication of this article.

\section{Acknowledgements}

We are grateful to Ms. Lynn Ayres M Ed. of the Tarnow Center for Self-Management for her final editing of our paper.

\section{Funding}

None.

\section{References}

1. Galderisi S, Sannita WG. Pharmaco-EEG: A history of progress and a missed opportunity. Clin EEG Neurosci. 2006;37(2):61-65.

2. Coburn KL, Lauterbach EC, Boutros NN, et al. The value of quantitative electroencephalography in clinical psychiatry: A report by the committee on research of the American Neuropsychiatric Association. J Neuropsychiatry Clin Neurosci. 2006;18(4):460-500.

3. Yamada T, Meng E. Practice guide for clinical neurophysiologic testing: EEG. Lippincott Williams \& Wilkins, Philadelphia, USA. 2009.

4. Asokan G, Pareja J, Niedermeyer E. Temporal minor slow and sharp EEG activity and cerebrovascular disorder. Clin Electroencephalogr . 1987;18(4):201-210.

5. Koberda JL, Moses A, Koberda P, et al. Clinical advantages of quantitative electroencephalogram (QEEG)- electrical neuroimaging application in general neurology practice. Clin EEG Neurosci. 2013;44(4):273-284.
6. Lakhan SE, Vieira K, Hamlat E. Biomarkers in psychiatry: Drawbacks and potential for misuse. Int Arch Med. 2010;3:1.

7. Bosl W, Tierney A, Tager-Flusberg H, et al. EEG complexity as a biomarker for autism spectrum disorder risk. BMC Med. 2011;9:18.

8. Vas G A, Cracco JB. Diffuse encephalopathies ( $2^{\text {nd }}$ edn), Current Practice of Clinical Electroencephalography. 1990. p.371-399.

9. Kaplan PW, Lesser RP. Long-term monitoring ( $2^{\text {nd }}$ edn $)$, Current Practice of Clinical Electroencephalography. 1990. p.513-534.

10. Gibbs FA, Gibbs EL. Atlas of electroencephalography. Cambridge, UK. 1941.

11. Neubrander J, Linden M, Gunkelman J, et al. QEEG-guided neurofeedback: New brain-based individualized evaluation and treatment for autism. The Journal of Austimone.2011;3(8):90-100.

12. Clarke AR, Barry RJ, McCarthy R, et al. Excess beta activity in children with attention-deficit/hyperactivity disorder: An atypical electrophysiological group. Psychiatry Res. 2001;103(2-3):205-218.

13. Shelley BP, Trimble MR, Boutros NN. Electroencephalographic cerebral dysrhythmic abnormalities in the trinity of nonepileptic general population, neuropsychiatric, and neurobehavioral disorders. $J$ Neuropsychiatry Clin Neurosci. 2006;20(1):7-22.

14. Peter HW, Stanley YS. Re sampling-based multiple testing: Examples and methods for p-value adjustment, John Wiley \& Sons. 1992.

15. Insel T. Director's Blog: Transforming Diagnosis. 2013.

16. Obama Barack. Executive Order - Improving Access to Mental Health Services for Veterans, Service Members and Military Families. White House. 2013. 